\title{
AÇÃO DE INSETICIDAS SOBRE OS OVOS E LAGARTAS DA BROCA-PEQUENA-DO- FRUTO DO TOMATE, EM BIOENSAIO DE LABORATÓRIO
}

\author{
S.A. De Bortoli ${ }^{1}$, S.R. Benvenga ${ }^{2}$, S. Gravena ${ }^{2}$, A.M. Vacari $^{1}$, H.X.L. Volpe ${ }^{1}$ \\ ${ }^{1}$ Universidade Estadual Paulista, Faculdade de Ciências Agrárias e Veterinárias, Departamento de \\ Fitossanidade, Via de Acesso Prof. Paulo Donato Castellane, s/no, CEP 14884-900, Jaboticabal, SP, Brasil.
}

\author{
RESUMO
}

\begin{abstract}
A broca-pequena-do-fruto é praga-chave na cultura do tomate por causar danos significativos às partes reprodutivas. Devido a isto, foi objetivo deste trabalho avaliar a eficiência de inseticidas sobre ovos e lagartas recém-emergidas, com e sem a adição de óleo vegetal (0,25\%), em bioensaios de laboratório. Frutos com ovos foram coletados em cultivo de tomate estaqueado na quinzena posterior à última aplicação de agrotóxicos, sendo selecionados os frutos com ovos de coloração variável de branco a marrom claro, com 1 e 4 dias de incubação, e imersos em $1 \mathrm{~L}$ da calda inseticida por 5 segundos. O delineamento estatístico utilizado foi o inteiramente casualizado, com média de 18 frutos nos tratamentos e de, aproximadamente, 4 ovos/fruto. A avaliação da ação inseticida de 24 produtos foi realizada após a imersão na calda inseticida, observando-se, sob microscópio estereoscópico, os ovos quanto à integridade do córion, textura e coloração, lagartas emergidas, bem como os orifícios de entrada e de saída das larvas (aos 7 e 21 dias). Os produtos testados diferiram da testemunha quanto à densidade de lagartas eclodidas, bem como quanto à redução populacional de lagartas, podendo-se destacar Trebon 100 SC (etofenprox; $200 \mathrm{~mL}$ do produto comercial/100 L), Lannate BR (methomil; 100 mL), Thiobel 500 (cartap; 250 g) e Vertimec 18 CE (abamectin; 100 mL). A adição de óleo vegetal resultou em incremento na eficiência dos produtos.

PALAVRAS-CHAVE: Neoleucinodes elegantalis, Lycopersicon esculentum, ovicida, lagarticida, tática de controle.
\end{abstract}

\begin{abstract}
ACTION OF INSECTICIDES ON TOMATO FRUIT BORER EGGS AND LARVAE USING LABORATORY BIOASSAY. The tomato fruit borer is a key tomato pest in light of its damages to the plants' reproductive parts. Therefore, the present study was aimed to evaluate the effectiveness of insecticides on the eggs and newly hatched larvae, when applied alone or associated with vegetable oil $(0.25 \%)$, in laboratory bioassays. Fruits with eggs were collected in staked tomato crops fifteen days after the last application of agro-chemicals, selecting the fruits with eggs colored from white to light brown, which had between 1 and 4 days of incubation, followed by immersion of the fruit for 5 seconds in $1 \mathrm{~L}$ of the insecticide solutions. The experimental design was completely randomized, with an average of 18 fruits per treatment ( 24 insecticides and a control) and about 4 eggs per fruit. The evaluations were performed 7 and 21 days after immersion, using a stereomicroscope to observe the corion integrity, egg color, number of larvae emerged, and larvae fruit holes (entry and exit). The insecticides differed significantly from the control, most notably Trebon SC 100 (etofenprox; $200 \mathrm{~mL}$ of the commercial product/100 L), Lannate BR (methomil; $100 \mathrm{~mL}$ ), Thiobel 500 (cartap; $250 \mathrm{~g}$ ) and Vertimec 18 CE (abamectin; $100 \mathrm{~mL}$ ). The addition of vegetable oil increased the insecticides' effectiveness.
\end{abstract}

KEY WORDS: Neoleucinodes elegantalis, Lycopersicon esculentum, ovicide, larvicide, control tactic.

\section{INTRODUÇÃO}

A broca-pequena-do-fruto, Neoleucinodes elegantalis (Guenée, 1854) (Lepidoptera: Crambidae), é considerada praga-chave na cultura do tomate. O inseto infesta as partes reprodutivas da planta e torna os frutos impróprios para o comércio e o processamento industrial, devido à destruição da polpa (GAllo et al., 2002; GravenA; BENVENGA, 2003). Os prejuízos podem chegar a até

${ }^{2}$ GRAVENA, Jaboticabal, SP, Brasil. 
90\% da produção (GALlo et al., 2002; MiRAnda et al., 2005).

As maiores populações da praga ocorrem durante o cultivo do verão (JoRDÃo; NAKANO, 2000; GRAVENA; Benvenga, 2003), em função da maior frequência das precipitações (NunEs; LEAL, 2001), época com condições de temperatura e de umidade relativa do ar favoráveis ao desenvolvimento de $N$. elegantalis (MARCANO, 1991a; 1991b).

Os adultos apresentam hábitos comportamentais de oviposição e cópula noturnos (MARCANO, 1991a; JAFFE et al., 2007), sendo, portanto, alvos biológicos não favoráveis para estudos de estratégias de controle químico da praga na cultura. As mariposas realizam a oviposição, predominantemente, na superfície dos frutos com diâmetro médio entre 2,3 e 2,5 cm (BLACKMER et al., 2001; ROdRIGUES FilHO et al., 2003; Gravena; Benvenga, 2003). O menor período de incubação encontrado por MARCANO (1991a) foi de 51 dias à temperatura de $20^{\circ} \mathrm{C}$, sendo, portanto, um alvo biológico favorável para a utilização de inseticidas com ação ovicida. GravenA; BENVENGA (2003) recomendaram adotar estratégias de seletividade ecológica e uso de inseticidas com seletividade fisiológica para complementar o controle biológico exercido por Trichogramma pretiosum (Hiley) (Hymenoptera: Trichogrammatidae) em cultivos com orientação de manejo.

Devido ao curto período de trânsito das lagartas na superfície dos frutos, o controle biológico naturalé de baixa eficiência enão superior a $2 \%$ de mortalidade da praga externamente ao fruto (PlazA et al., 1992). Nesse sentido, trabalhos que avaliam a eficiência dos inseticidas sobre os ovos e lagartas recém-eclodidas, por serem as fases de maior vulnerabilidade da praga, são de suma importância para orientar a rotação de produtos em programas de manejo. Por outro lado, os inseticidas quando associados com adjuvantes podem incrementar a eficiência de controlee garantir melhor qualidade da aplicação, contribuindo para a redução da intensidade de aplicações com benefícios ecológicos, econômicos e sociais.

Assim, o presente trabalho teve por objetivo avaliar a eficiência de inseticidas de diferentes grupos químicos sobre os ovos e lagartas de $N$. elegantalis recém-emergidas, quando aplicados deforma isolada ou associados com um adjuvante, em bioensaios de laboratório.

\section{MATERIAL E MÉTODOS}

Frutos com ovos de N. elegantalis foram coletados em cultivos comerciais de tomate, Lycopersicon esculentum, híbrido Alambra, em sistema estaqueado de condução, no Município de Monte Mor, SP, na quinzena posterior à última aplicação de inseticidas.
Foram selecionados frutos que apresentavam os ovos depositados em sua superfície e acondicionados em cartelas de papelão para o transporte até ao laboratório, onde foram observados sob microscópio estereoscópico, sendo utilizados nos bioensaios aqueles de coloração variável de branco a marrom claro que, segundo MuÑoz et al. (1991), apresentam entre 1 e 4 dias de incubação. Quantificou-se o número de ovos por postura estabelecendo distribuição uniforme entre os tratamentos. Para os bioensaios de aplicação isolada foram utilizados, em média, 18 frutos infestados com 4,2 $\pm 0,1$ ovos, totalizando=se aproximadamente 75 ovos por tratamento. Nos bioensaios com a adição de óleo vegetal $(0,25 \%)$ foram utilizados, em média, também 18 frutos, porém, com 4,3 $\pm 0,1$ ovos, resultando um valor aproximado de 77 ovos por tratamento. O delineamento foi inteiramente casualizado e os tratamentos empregados estão descritos na Tabela 1. As informações apresentadas sobre os produtos foram baseadas no Agrofit (BRASIL, 2010).

Os tratamentos foram aplicados por imersão individual de frutos na calda inseticida e no tratamento inseticida em associação com o óleo vegetal, porcerca de 5 segundos. Para a secagem, os ovos foram mantidos em placas de Petri sobre bancada no interior do laboratório. Os frutos com o resíduo seco foram mantidos em sala climatizada com temperatura de $25 \pm 2^{\circ} \mathrm{C}$ e umidade relativa do ar de $70 \pm 10 \%$.

A avaliação da ação dos inseticidas sobre os ovos foi realizada 7 dias após a imersão, sendo os frutos individualmente analisados no estereomicroscópio. Inicialmente, foi feita uma análise sobre a integridade do córion e o aspecto geral quanto à textura e à coloração. Foram considerados de ação ovicida, os inseticidas cujo córion dos ovos submetidos aos tratamentos não apresentaram qualquer sinal de raspagem pela lagarta para o rompimento e eclosão em direção à superfície do fruto. Também foram incluídos como ação ovicida os produtos cujos ovos apresentavam aspecto gelatinoso, disformes e sem qualquer indício da formação do embrião.

A avaliação da ação dos inseticidas sobre as larvas recém-eclodidas foi feita diariamente com a observação dos frutos sob microscópio estereoscópico até 7 dias após a imersão dos frutos. Foram considerados como de ação lagarticida os produtos cujo ovo apresentava a lagarta completamente desenvolvida e o córion com sinais de raspagem, porém, sem que a lagarta tivesse conseguido atingir o meio externo, além daqueles onde era claramente visível o orifício de saída da lagarta, bem com ela imóvel próxima ao córion. Por fim, o efeito de choque também foi estendido para os casos em que havia sinais primários de alimentação no epicarpo, mas sem que a entrada no fruto fosse concluída. 
Tabela 1 - Tratamentos testados sobre os ovos de Neoleucinodes elegantalis, em frutos do tomateiro, em condições de laboratório.

\begin{tabular}{|c|c|c|c|c|}
\hline \multicolumn{5}{|c|}{ Tratamentos } \\
\hline Classe & Inseticida & Ingrediente ativo (concentração) & Grupo químico & $\begin{array}{l}\text { Dose P.C. (100 L) } \\
\text { aplicação isolada e } \\
\text { óleo vegetal } 0,25 \%\end{array}$ \\
\hline $\mathrm{B}$ & Atabron $50 \mathrm{CE}$ & chlorfluazuron (50 g/L) & benzoilureia & $100 \mathrm{~mL}$ \\
\hline $\mathrm{C}$ & Cascade 100 & flufenoxuron (100 g/L) & benzoilureia & $50 \mathrm{~mL}$ \\
\hline $\mathrm{B}$ & Certero & triflumuron $(480 \mathrm{~g} / \mathrm{L})$ & benzoilureia & $30 \mathrm{~mL}$ \\
\hline B & Gallaxy $100 \mathrm{CE}$ & novaluron $(100 \mathrm{~g} / \mathrm{L})$ & benzoilureia & $25 \mathrm{~mL}$ \\
\hline A, B & Match CE & lufenuron $(50 \mathrm{~g} / \mathrm{L})$ & acilureias & $100 \mathrm{~mL}$ \\
\hline A, B & Intrepid 240 SC & methoxyfenozide (240 g/L) & hidrazida & $50 \mathrm{~mL}$ \\
\hline A, B & Rumo GDA & indoxacarb $(300 \mathrm{~g} / \mathrm{kg})$ & oxadiazina & $10 \mathrm{~g}$ \\
\hline A, B & Akito & betacypermethrin (100 g/L) & piretroide & $50 \mathrm{~mL}$ \\
\hline A, B & Meothrin 300 & fenpropathrin $(300 \mathrm{~g} / \mathrm{L})$ & piretroide & $50 \mathrm{~mL}$ \\
\hline A & Stallion 60 CS & gamacyhalothrin $(60 \mathrm{~g} / \mathrm{L})$ & piretroide & $15 \mathrm{~mL}$ \\
\hline A, B & Trebon 100 SC & etofenprox $(100 \mathrm{~g} / \mathrm{L})$ & éter difenílico & $200 \mathrm{~mL}$ \\
\hline A, B & Lorsban 480 BR & clorpyrifos (480 g/L) & fosforado & $150 \mathrm{~mL}$ \\
\hline $\mathrm{B}$ & Orthene 750 BR & acephato $(750 \mathrm{~g} / \mathrm{kg})$ & fosforado & $100 \mathrm{~g}$ \\
\hline A, B & Lannate BR & methomil $(215 \mathrm{~g} / \mathrm{L})$ & metilcarbamato & $100 \mathrm{~mL}$ \\
\hline $\mathrm{C}$ & Deltaphos EC & $\begin{array}{c}\text { deltamethrin }(10 \mathrm{~g} / \mathrm{L})+ \\
\text { triazophos }(350 \mathrm{~g} / \mathrm{L})\end{array}$ & piretroide + fosforado & $100 \mathrm{~mL}$ \\
\hline$A, B$ & Thiobel 500 & cartap $(500 \mathrm{~g} / \mathrm{kg})$ & tiocarbamato & $250 \mathrm{~g}$ \\
\hline B & Vertimec 18 CE & abamectin $(18 \mathrm{~g} / \mathrm{L})$ & avermectina & $100 \mathrm{~mL}$ \\
\hline B & Cordial 100 & pyriproxifen (100 g/L) & piridil éter & $75 \mathrm{~mL}$ \\
\hline B & Tedion 80 & tetradifon $(80 \mathrm{~g} / \mathrm{L})$ & clorodifenilsulfon & $300 \mathrm{~mL}$ \\
\hline B & Polo 500 PM & diafenthiuron $(500 \mathrm{~g} / \mathrm{kg})$ & feniltioureia & $50 \mathrm{~g}$ \\
\hline B & Pirate & chlorfenapyr $(240 \mathrm{~g} / \mathrm{L})$ & análogo pirazol & $50 \mathrm{~mL}$ \\
\hline B & Oberon & spiromesifen $(240 \mathrm{~g} / \mathrm{L})$ & cetoenol & $50 \mathrm{~mL}$ \\
\hline $\mathrm{C}$ & Belt & flubendiamid $(480 \mathrm{~g} / \mathrm{L})$ & ác. aminophtalico & $15 \mathrm{~mL}$ \\
\hline A, B & Agree & B. thuringiensis (500 g/ kg) & biológico & $100 \mathrm{~g}$ \\
\hline \multicolumn{2}{|c|}{ Testemunha (água) } & água & $X x$ & $X x$ \\
\hline \multicolumn{2}{|c|}{ Testemunha (Naturalóleo) } & óleo vegetal (930 mL/L) & hidrocarboneto & $250 \mathrm{~mL}$ \\
\hline
\end{tabular}

A - Produto registrado para a cultura do tomate e indicado para Neoleucinodes elegantalis;

B - Produto registrado para a cultura do tomate e indicado para outra praga chave;

C - Produto em fase de registro para a cultura do tomate ou indicado para outra cultura.

Foi realizada uma avaliação final 21 dias após a imersão dos frutos na calda inseticida. Nessa avaliação o alvo biológico foi a lagarta, porém, neste caso, em fase de crescimento um pouco mais adiantada. $O$ ciclo de desenvolvimento das lagartas nos frutos foi avaliado quanto à presença de orifícios de saída nos frutos. Uma avaliação complementar foi realizada com o auxílio de um estilete, fazendo-se cortes nos frutos para a verificação, ou não, da presença de lagartas.

Os dados obtidos foram submetidos à análise de variância pelo teste $\mathrm{F}$ e as médias foram comparadas pelo teste de Tukey. Para o cálculo da eficiência dos tratamentos, quanto à ação ovicida, foi utilizada a fórmula de Аввотт (1925).

\section{RESULTADOS}

Eficiência de controle/ação ovicida - A eficiência dosinseticidas em aplicação isolada e adicionados de óleo vegetal $(0,25 \%)$ na inviabilidade dos ovos está expressa na Figura 1. Os produtos com destacada ação ovicida em aplicação isolada e adicionados de óleo vegetal $(0,25 \%)$ foram o Trebon $100 \mathrm{SC}(200 \mathrm{~mL})$, Lannate BR (100 mL), Thiobel 500 (250 g) e Vertimec 18 CE (100 mL). A adição de óleo resultou ganho significativo na eficiência de ação ovicida (Figura 1) destes produtos que atingiram valores da ordem de $64,52,64$ e $67 \%$, respectivamente.

Densidade de lagartas eclodidas - A análise da integridade do córion, textura e coloração indicou que Trebon 100 SC (200 mL/100 L), em aplicação isolada, diferiu significativamente da testemunha quanto à densidade de lagartas eclodidas (Tabela 2), caracterizando-se por apresentar ação ovicida. $\mathrm{O}$ mesmo resultado foi verificado para Lannate BR (100 $\mathrm{mL})$, Thiobel 500 (250 g) e Vertimec 18 CE (100 mL), não diferindo significativamente entre si.

Com a adição de óleo vegetal, verificou-se ação ovicida para o Trebon 100 SC (200 mL), Lannate BR (100 mL), Thiobel 500 (250 g) e Vertimec 18 CE (100 
$\mathrm{mL})$, por resultar em número médio de lagartas eclodidas significativamente inferiores ao ocorridona testemunha. O número médio de lagartas eclodidas foi da ordem de 1,1 $\pm 0,2$ e 1,6 \pm 0,5 insetos/fruto, respectivamente, para o Vertimec $18 \mathrm{CE}(100 \mathrm{~mL}) \mathrm{e}$ Lannate BR (100 mL), não diferindo significativamente entre si. $\mathrm{Na}$ testemunha foram observadas 3,3 \pm 0,5 lagartas eclodidas/fruto.

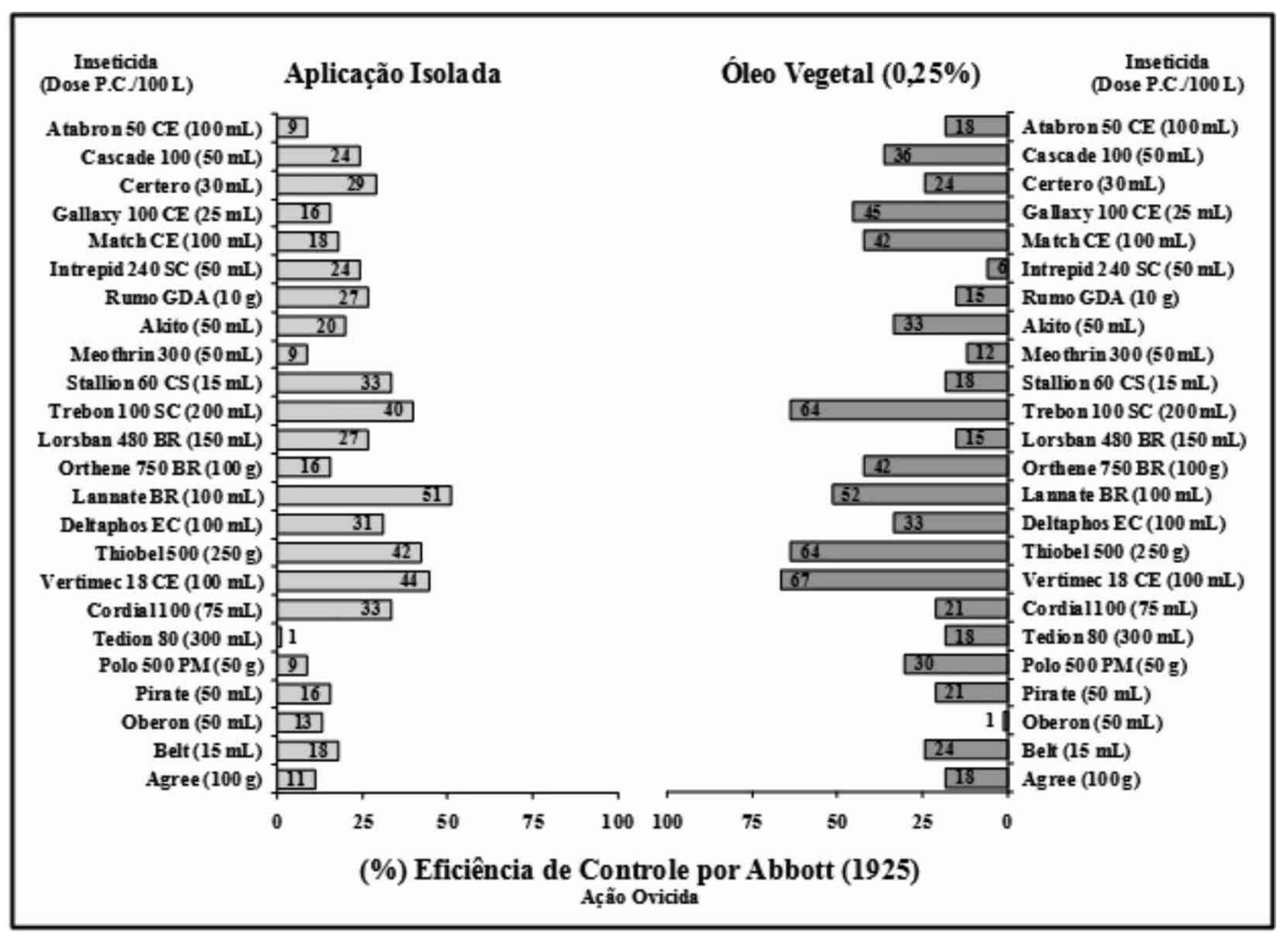

Fig. 1 - Eficiência dos inseticidas sobre os ovos de Neoleucinodes elegantalis em frutos do tomateiro, submetidos ao controle químico em bioensaio de laboratório.

Considerando-se o número médio de lagartas eclodidas verificou-se que a ação ovicida dos inseticidas adicionados de óleo vegetal (0,25\%) foi significativamente superior em relação aos produtos em aplicação isolada (Tabela 2), com valores de 3,5 \pm 0,1 e 2,3 \pm 0,1 lagartas/fruto, respectivamente. A ação ovicida de Trebon 100 SC, Lannate BR, Thiobel 500 e Vertimec 18 CE foi mais pronunciada quando adicionados de óleo vegetal $(0,25 \%)$.

Tabela 2 - Densidade de lagartas de Neoleucinodes elegantalis eclodidas em frutos do tomateiro, submetidos ao controle químico em bioensaio de laboratório.

\begin{tabular}{|c|c|c|c|c|c|c|c|}
\hline \multicolumn{8}{|c|}{ Densidade de lagartas eclodidas $^{1 / 2}$} \\
\hline \multirow{2}{*}{ Inseticida } & \multirow{2}{*}{ Dose (100 L) } & \multicolumn{3}{|c|}{ Aplicação isolada } & \multicolumn{3}{|c|}{ Óleo vegetal $(0,25 \%)$} \\
\hline & & (n) & Média : & & (n) & Média \pm & \\
\hline Atabron $50 \mathrm{CE}$ & $100 \mathrm{~mL}$ & 18 & $4,1 \pm 0,4$ & $\mathrm{Aab}$ & 19 & $2,7 \pm 0,5$ & $\mathrm{~B} \mathrm{ab}$ \\
\hline Cascade 100 & $50 \mathrm{~mL}$ & 18 & $3,4 \pm 0,7$ & $\mathrm{~A} a \mathrm{~b}$ & 17 & $2,1 \pm 0,6$ & $\mathrm{~A} a b$ \\
\hline Certero & $30 \mathrm{~mL}$ & 20 & $3,2 \pm 0,3$ & $\mathrm{~A} a \mathrm{~b}$ & 15 & $2,5 \pm 0,4$ & $\mathrm{~B} a b$ \\
\hline Gallaxy $100 \mathrm{CE}$ & $25 \mathrm{~mL}$ & 18 & $3,8 \pm 0,6$ & $\mathrm{~A} a \mathrm{~b}$ & 19 & $1,8 \pm 0,5$ & $\mathrm{~B} a b$ \\
\hline Match CE & $100 \mathrm{~mL}$ & 18 & $3,7 \pm 0,8$ & $\mathrm{~A} a \mathrm{~b}$ & 18 & $1,9 \pm 0,4$ & $\mathrm{~B} a b$ \\
\hline Intrepid 240 SC & $50 \mathrm{~mL}$ & 19 & $3,4 \pm 0,5$ & $\mathrm{~A} a \mathrm{~b}$ & 18 & $3,1 \pm 0,5$ & $\mathrm{~A} a b$ \\
\hline Rumo GDA & $10 \mathrm{~g}$ & 21 & $3,3 \pm 0,6$ & $\mathrm{~A} a b$ & 16 & $2,8 \pm 0,7$ & $\mathrm{~A} a b$ \\
\hline Akito & $50 \mathrm{~mL}$ & 18 & $3,6 \pm 0,3$ & $\mathrm{~A} a \mathrm{~b}$ & 22 & $2,2 \pm 0,3$ & $\mathrm{~A} a b$ \\
\hline Meothrin 300 & $50 \mathrm{~mL}$ & 16 & $4,1 \pm 0,6$ & $\mathrm{~A} a b$ & 19 & $2,9 \pm 0,4$ & $\mathrm{~A} a b$ \\
\hline Stallion 60 CS & $15 \mathrm{~mL}$ & 21 & $3,0 \pm 0,4$ & $\mathrm{~A} a \mathrm{~b}$ & 15 & $2,7 \pm 0,6$ & $\mathrm{~A} a b$ \\
\hline Trebon 100 SC & $200 \mathrm{~mL}$ & 18 & $2,7 \pm 0,5$ & A b & 19 & $1,2 \pm 0,3$ & $\mathrm{~B} b$ \\
\hline
\end{tabular}


Tabela 2 - Continuação

\begin{tabular}{|c|c|c|c|c|c|c|c|}
\hline \multicolumn{8}{|c|}{ Densidade de lagartas eclodidas ${ }^{1 / 2}$} \\
\hline \multirow{2}{*}{ Inseticida } & \multirow{2}{*}{ Dose $(100 \mathrm{~L})$} & \multicolumn{3}{|c|}{ Aplicação isolada } & \multicolumn{3}{|c|}{ Óleo vegetal $(0,25 \%)$} \\
\hline & & (n) & Média \pm EF & & (n) & Média \pm EP & \\
\hline Lorsban 480 BR & $150 \mathrm{~mL}$ & 20 & $3,3 \pm 0,4$ & $\mathrm{~A} a \mathrm{~b}$ & 18 & $2,8 \pm 0,8$ & $\mathrm{Aab}$ \\
\hline Orthene 750 BR & $100 \mathrm{~g}$ & 18 & $3,8 \pm 0,6$ & $\mathrm{~A} a \mathrm{~b}$ & 19 & $1,9 \pm 0,4$ & $\mathrm{~B} a b$ \\
\hline Lannate BR & $100 \mathrm{~mL}$ & 21 & $2,2 \pm 0,4$ & A b & 13 & $1,6 \pm 0,5$ & B b \\
\hline Deltaphos EC & $100 \mathrm{~mL}$ & 17 & $3,1 \pm 0,7$ & $\mathrm{~A} a \mathrm{~b}$ & 19 & $2,2 \pm 0,4$ & $\mathrm{~A} a b$ \\
\hline Thiobel 500 & $250 \mathrm{~g}$ & 16 & $2,6 \pm 0,5$ & A b & 18 & $1,2 \pm 0,2$ & B b \\
\hline Vertimec 18 CE & $100 \mathrm{~mL}$ & 18 & $2,5 \pm 0,4$ & A b & 22 & $1,1 \pm 0,2$ & B b \\
\hline Cordial 100 & $75 \mathrm{~mL}$ & 21 & $3,0 \pm 0,5$ & $\mathrm{~A} a b$ & 18 & $2,6 \pm 0,6$ & $\mathrm{~A} a b$ \\
\hline Tedion 80 & $300 \mathrm{~mL}$ & 16 & $4,9 \pm 0,8$ & $\mathrm{~A} a b$ & 19 & $2,7 \pm 0,5$ & $\mathrm{~A} a b$ \\
\hline Polo 500 PM & $50 \mathrm{~g}$ & 18 & $4,1 \pm 0,5$ & $\mathrm{~A} a b$ & 19 & $2,3 \pm 0,5$ & $\mathrm{~B} a b$ \\
\hline Pirate & $50 \mathrm{~mL}$ & 18 & $3,8 \pm 0,7$ & $\mathrm{~A} a b$ & 15 & $2,6 \pm 0,4$ & $\mathrm{~A} a b$ \\
\hline Oberon & $50 \mathrm{~mL}$ & 17 & $3,9 \pm 0,5$ & $\mathrm{~A} a \mathrm{~b}$ & 15 & $3,5 \pm 0,7$ & $\mathrm{~A} a b$ \\
\hline Belt & $15 \mathrm{~mL}$ & 18 & $3,7 \pm 0,6$ & $\mathrm{~A} a b$ & 19 & $2,5 \pm 0,5$ & $\mathrm{~A} a b$ \\
\hline Agree & $100 \mathrm{~g}$ & 11 & $4,0 \pm 0,9$ & $\mathrm{~A} a \mathrm{~b}$ & 11 & $2,7 \pm 0,6$ & $\mathrm{~B} a b$ \\
\hline Testemunha & & 22 & $4,5 \pm 0,5$ & $\mathrm{~A} \mathrm{a}$ & 21 & $3,3 \pm 0,5$ & $\mathrm{~B}$ a \\
\hline Média & & 18 & $3,5 \pm 0,1$ & A & 18 & $2,3 \pm 0,1$ & B \\
\hline
\end{tabular}

${ }^{1}$ Dados originais. Para a análise estatística foram transformados em SQRT $(x+0,5)$.

${ }^{2}$ Nas linhas, médias seguidas de mesma letra maiúscula, e nas colunas, seguidas de mesma letra minúscula, não diferem entre si por Tukey $(\mathrm{P} \leq 0,05)$.

Tabela 3 - Densidade de orifícios de entrada de lagartas de Neoleucinodes elegantalis, em frutos do tomateiro, submetidos ao controle químico em bioensaio de laboratório.

\begin{tabular}{|c|c|c|c|c|c|c|c|}
\hline \multicolumn{8}{|c|}{ Densidade de orifícios de entrada $^{1 / 2}$} \\
\hline \multirow{2}{*}{ Inseticida } & \multirow[b]{2}{*}{ Dose (100 L) } & \multicolumn{3}{|c|}{ Aplicação isolada } & \multicolumn{3}{|c|}{ Óleo vegetal $(0,25 \%)$} \\
\hline & & (n) & \multicolumn{2}{|c|}{ Média \pm EP } & (n) & \multicolumn{2}{|c|}{ Média \pm EP } \\
\hline Atabron $50 \mathrm{CE}$ & $100 \mathrm{~mL}$ & 18 & $3,3 \pm 0,5$ & $\mathrm{~A} a \mathrm{~b}$ & 19 & $2,5 \pm 0,4$ & $\mathrm{~A}$ a b c \\
\hline Cascade 100 & $50 \mathrm{~mL}$ & 18 & $2,9 \pm 0,6$ & $\mathrm{~A} a \mathrm{bcd}$ e & 17 & $1,4 \pm 0,4$ & B bcde $\mathrm{fgh}$ \\
\hline Certero & $30 \mathrm{~mL}$ & 20 & $2,5 \pm 0,3$ & $\mathrm{~A} a \mathrm{bcd}$ & 15 & $1,7 \pm 0,5$ & B bcdef $\mathrm{gh}$ \\
\hline Gallaxy $100 \mathrm{CE}$ & $25 \mathrm{~mL}$ & 18 & $2,9 \pm 0,5$ & $\mathrm{~A} a \mathrm{bcd}$ & 19 & $1,4 \pm 0,4$ & B b d e f g h \\
\hline Match CE & $100 \mathrm{~mL}$ & 18 & $3,2 \pm 0,7$ & $\mathrm{~A} a \mathrm{bc}$ & 18 & $1,6 \pm 0,4$ & B bcdef $\mathrm{gh}$ \\
\hline Intrepid 240 SC & $50 \mathrm{~mL}$ & 19 & $2,7 \pm 0,5$ & $\mathrm{~A} a \mathrm{bcd} \mathrm{e}$ & 18 & $2,8 \pm 0,5$ & $\mathrm{~A} a \mathrm{~b}$ \\
\hline Rumo GDA & $10 \mathrm{~g}$ & 21 & $2,4 \pm 0,5$ & A b d e & 16 & $1,4 \pm 0,3$ & B b d e f g h \\
\hline Akito & $50 \mathrm{~mL}$ & 18 & $1,2 \pm 0,4$ & $\mathrm{c} \mathrm{de} \mathrm{f} g$ & 22 & $0,5 \pm 0,2$ & e $\mathrm{f} \mathrm{gh}$ \\
\hline Meothrin 300 & $50 \mathrm{~mL}$ & 16 & $2,6 \pm 0,6$ & $\mathrm{~A} \mathrm{a} \mathrm{bcdef}$ & 19 & $1,8 \pm 0,3$ & A a b c d e f g \\
\hline Stallion 60 CS & $15 \mathrm{~mL}$ & 21 & $0,3 \pm 0,2$ & A $\quad \mathrm{g}$ & 15 & $0,9 \pm 0,3$ & $c d e f g h$ \\
\hline Trebon 100 SC & $200 \mathrm{~mL}$ & 18 & $0,6 \pm 0,2$ & $\mathrm{f} \mathrm{g}$ & 19 & $0,5 \pm 0,3$ & $\mathrm{~g} \mathrm{~h}$ \\
\hline Lorsban $480 \mathrm{BR}$ & $150 \mathrm{~mL}$ & 20 & $1,0 \pm 0,2$ & c d e $\mathrm{f} g$ & 18 & $0,7 \pm 0,3$ & $\mathrm{~d}$ e $\mathrm{f} \mathrm{g} \mathrm{h}$ \\
\hline Orthene 750 BR & $100 \mathrm{~g}$ & 18 & $1,5 \pm 0,4$ & A $b c d e f g$ & 19 & $1,4 \pm 0,3$ & A b d e f $g h$ \\
\hline Lannate BR & $100 \mathrm{~mL}$ & 21 & $0,7 \pm 0,2$ & e $f \mathrm{~g}$ & 13 & $0,5 \pm 0,2$ & $\mathrm{~g} \mathrm{~h}$ \\
\hline Deltaphos EC & $100 \mathrm{~mL}$ & 17 & $1,0 \pm 0,4$ & $c d e f g$ & 19 & $0,5 \pm 0,2$ & $\mathrm{~g} \mathrm{~h}$ \\
\hline Thiobel 500 & $250 \mathrm{~g}$ & 16 & $0,6 \pm 0,2$ & $f \mathrm{~g}$ & 18 & $0,1 \pm 0,1$ & B \\
\hline Vertimec 18 CE & $100 \mathrm{~mL}$ & 18 & $0,9 \pm 0,3$ & $\mathrm{~d}$ e $\mathrm{f} \mathrm{g}$ & 22 & $0,5 \pm 0,2$ & $\mathrm{f} \mathrm{g} \mathrm{h}$ \\
\hline Cordial 100 & $75 \mathrm{~mL}$ & 21 & $2,0 \pm 0,4$ & A $\quad b c d e f$ & 18 & $1,9 \pm 0,5$ & $A a b c d e f g$ \\
\hline Tedion 80 & $300 \mathrm{~mL}$ & 16 & $4,0 \pm 0,7$ & $\mathrm{~A} a \mathrm{~b}$ & 19 & $2,2 \pm 0,3$ & A a b c d e \\
\hline Polo 500 PM & $50 \mathrm{~g}$ & 18 & $3,3 \pm 0,5$ & $\mathrm{~A} a \mathrm{~b}$ & 19 & $2,1 \pm 0,4$ & $\mathrm{~A} a \mathrm{bcd}$ e $\mathrm{f}$ \\
\hline Pirate & $50 \mathrm{~mL}$ & 18 & $2,3 \pm 0,4$ & A b c d e f & 15 & $1,7 \pm 0,4$ & A b c d e $f g h$ \\
\hline Oberon & $50 \mathrm{~mL}$ & 17 & $3,5 \pm 0,4$ & $\mathrm{~A} a \mathrm{~b}$ & 15 & $3,0 \pm 0,7$ & $A a b c d$ \\
\hline Belt & $15 \mathrm{~mL}$ & 18 & $0,9 \pm 0,2$ & A $\quad c d e f g$ & 19 & $0,8 \pm 0,2$ & A $\quad c d e f g h$ \\
\hline Agree & $100 \mathrm{~g}$ & 11 & $2,6 \pm 0,8$ & $\mathrm{~A} a \mathrm{bcde}$ & 11 & $1,5 \pm 0,3$ & B bcdef gh \\
\hline Testemunha & & 22 & $4,1 \pm 0,5$ & $\mathrm{~A}$ a & 21 & $3,0 \pm 0,5$ & $\mathrm{~B}$ a \\
\hline Média & & 18 & $2,1 \pm 0,1$ & A & 18 & $1,4 \pm 0,1$ & B \\
\hline
\end{tabular}

${ }^{1}$ Dados originais. Para a análise estatística foram transformados em SQRT $(x+0,5)$.

${ }^{2}$ Nas linhas, médias seguidas de mesma letra maiúscula, e nas colunas, seguidas de mesma letra minúscula, não diferem entre si por Tukey $(\mathrm{P} \leq 0,05)$. 
Densidade de orifícios de entrada - Quanto à densidade de orifícios de entrada na superfície dos frutos (Tabela 3), os produtos Rumo GDA (10 g/100 L), Akito (50 mL), Stallion 60 CS (15 mL), Trebon 100 SC (200 mL), Lorsban 480 BR (150 mL), Orthene 750 BR (100 g), Lannate BR (100 mL), Deltaphos EC (100 $\mathrm{mL})$, Thiobel $500(250 \mathrm{~g})$, Vertimec $18 \mathrm{CE}(100 \mathrm{~mL})$, Pirate $(50 \mathrm{~mL})$ e Belt $(15 \mathrm{~mL})$, em aplicação isolada e adicionados de óleo vegetal $(0,25 \%)$, diferiram estatisticamente da testemunha, caracterizando-se por apresentarem ação sobre lagartas recém-eclodidas. Rumo GDA e Thiobel 500 diferiram estatisticamente entre si nos dois modos de aplicação, com resultado de controle mais efetivo em associação com óleo vegetal (Tabela 3). O mesmo resultado quando adi- cionado óleo vegetal foi, verificado para o Cascade 100 (50 mL), Certero (30 mL), Gallaxy 100 CE (25 $\mathrm{mL})$, Match CE (100 mL) e Agree (100 g), diferindo entre si quando comparados nos dois métodos de aplicação.

Na testemunha, os números médios de lagartas que perfuraram os frutos tratados somente com e sem a adição de óleo vegetal $(0,25 \%)$ foi da ordem de $4,1 \pm 0,5$ e $3,0 \pm 0,5$ insetos/fruto, respectivamente, indicando que o adjuvante contribui positivamente no controle de lagartas recém-eclodidas. $\mathrm{O}$ aumento na eficiência de controle pode ser aferido também pelo número médio de orifícios de entrada, com valores da ordem de 2,1 $\pm 0,1$ e de 1,4 $\pm 0,1$ por fruto, respectivamente com e sem óleo.

Tabela 4 - Densidade de orifícios de saída de lagartas de Neoleucinodes elegantalis, em frutos do tomateiro, submetidos ao controle químico em bioensaio de laboratório.

\begin{tabular}{|c|c|c|c|c|c|c|c|c|c|}
\hline \multicolumn{10}{|c|}{ Densidade de orifícios de saída ${ }^{1 / 2}$} \\
\hline \multirow{3}{*}{$\begin{array}{l}\text { Inseticida } \\
\text { Atabron } 50 \mathrm{CE}\end{array}$} & \multirow{3}{*}{$\frac{\text { Dose }(100 \mathrm{~L})}{100 \mathrm{~mL}}$} & \multicolumn{4}{|c|}{ Aplicação isolada } & \multicolumn{4}{|c|}{ Óleo vegetal $(0,25 \%)$} \\
\hline & & \multirow{2}{*}{$\frac{(\mathrm{n})}{18}$} & \multicolumn{3}{|c|}{ Média \pm EP } & \multirow{2}{*}{$\frac{(\mathrm{n})}{19}$} & \multicolumn{3}{|c|}{ Média \pm EP } \\
\hline & & & $2,4 \pm 0,3$ & A & $\mathrm{bcd}$ & & $1,3 \pm 0,2$ & $\mathrm{~B}$ & bcde \\
\hline Cascade 100 & $50 \mathrm{~mL}$ & 18 & $2,4 \pm 0,5$ & A & bcde & 17 & $1,2 \pm 0,4$ & $\mathrm{~A}$ & bcdefg \\
\hline Certero & $30 \mathrm{~mL}$ & 20 & $2,3 \pm 0,3$ & A & $\mathrm{bcd}$ & 15 & $1,5 \pm 0,5$ & $\mathrm{~B}$ & bcdefg \\
\hline Gallaxy $100 \mathrm{CE}$ & $25 \mathrm{~mL}$ & 18 & $2,4 \pm 0,4$ & A & $\mathrm{bcd}$ & 19 & $1,3 \pm 0,3$ & B & bcdefg \\
\hline Match CE & $100 \mathrm{~mL}$ & 18 & $2,0 \pm 0,4$ & A & bcdef & 18 & $1,1 \pm 0,3$ & B & bcdefg \\
\hline Intrepid 240 SC & $50 \mathrm{~mL}$ & 19 & $1,9 \pm 0,4$ & A & bcdefg & 18 & $1,2 \pm 0,2$ & A & bcdef \\
\hline Rumo GDA & $10 \mathrm{~g}$ & 21 & $1,3 \pm 0,4$ & A & cdefghi & 16 & $0,6 \pm 0,2$ & A & cdefg \\
\hline Akito & $50 \mathrm{~mL}$ & 18 & $0,9 \pm 0,3$ & A & defghi & 22 & $0,5 \pm 0,2$ & A & defg \\
\hline Meothrin 300 & $50 \mathrm{~mL}$ & 16 & $2,1 \pm 0,4$ & A & bcdefg & 19 & $1,1 \pm 0,2$ & $\mathrm{~B}$ & bcdefg \\
\hline Stallion 60 CS & $15 \mathrm{~mL}$ & 21 & $0,05 \pm 0,1$ & B & $\mathrm{i}$ & 15 & $0,8 \pm 0,3$ & A & bcdefg \\
\hline Trebon 100 SC & $200 \mathrm{~mL}$ & 18 & $0,5 \pm 0,2$ & A & ghi & 19 & $0,5 \pm 0,2$ & A & efg \\
\hline Lorsban $480 \mathrm{BR}$ & $150 \mathrm{~mL}$ & 20 & $0,5 \pm 0,2$ & A & fghi & 18 & $0,2 \pm 0,1$ & A & ef $g$ \\
\hline Orthene 750 BR & $100 \mathrm{~g}$ & 18 & $1,2 \pm 0,3$ & A & cdefghi & 19 & $1,0 \pm 0,3$ & A & bcdefg \\
\hline Lannate BR & $100 \mathrm{~mL}$ & 21 & $0,5 \pm 0,2$ & A & fghi & 13 & $0,5 \pm 0,2$ & A & e $f g$ \\
\hline Deltaphos EC & $100 \mathrm{~mL}$ & 17 & $0,2 \pm 0,1$ & A & $\mathrm{hi}$ & 19 & $0,1 \pm 0,1$ & A & g \\
\hline Thiobel 500 & $250 \mathrm{~g}$ & 16 & $0,3 \pm 0,1$ & A & hi & 18 & $0,1 \pm 0,1$ & A & $\mathrm{g}$ \\
\hline Vertimec $18 \mathrm{CE}$ & $100 \mathrm{~mL}$ & 18 & $0,2 \pm 0,1$ & A & $\mathrm{hi}$ & 22 & $0,1 \pm 0,1$ & A & $\mathrm{fg}$ \\
\hline Cordial 100 & $75 \mathrm{~mL}$ & 21 & $1,4 \pm 0,3$ & A & bcdefgh & 18 & $1,3 \pm 0,4$ & A & bcdef \\
\hline Tedion 80 & $300 \mathrm{~mL}$ & 16 & $3,8 \pm 0,7$ & A & & 19 & $1,8 \pm 0,3$ & & $b c d$ \\
\hline Polo 500 PM & $50 \mathrm{~g}$ & 18 & $2,6 \pm 0,4$ & & $\mathrm{bc}$ & 19 & $1,8 \pm 0,3$ & & $\mathrm{bc}$ \\
\hline Pirate & $50 \mathrm{~mL}$ & 18 & $1,8 \pm 0,3$ & $\mathrm{~A}$ & bcdefg & 15 & $1,1 \pm 0,3$ & B & bcdefg \\
\hline Oberon & $50 \mathrm{~mL}$ & 17 & $3,1 \pm 0,4$ & A & bc & 15 & $2,6 \pm 0,6$ & & \\
\hline Belt & $15 \mathrm{~mL}$ & 18 & $0,2 \pm 0,2$ & A & hi & 19 & $0,2 \pm 0,1$ & A & efg \\
\hline Agree & $100 \mathrm{~g}$ & 11 & $2,5 \pm 0,7$ & A & $\mathrm{bcd}$ & 11 & $1,2 \pm 0,3$ & $\mathrm{~B}$ & bcdefg \\
\hline Testemunha & & 22 & $3,9 \pm 0,5$ & A & & 21 & $2,7 \pm 0,4$ & $\mathrm{~B}$ & \\
\hline Média & & 18 & $1,5 \pm 0,1$ & A & & 18 & $1,0 \pm 0,1$ & B & \\
\hline
\end{tabular}

${ }^{1}$ Dados originais. Para a análise estatística foram transformados em SQRT $(x+0,5)$.

${ }^{2}$ Nas linhas, médias seguidas de mesma letra maiúscula, e nas colunas, seguidas de mesma letra minúscula, não diferem entre si por Tukey $(\mathrm{P} \leq 0,05)$. 
Densidade de orifícios de saída - A Tabela 4 mostra os resultados dos tratamentos no desenvolvimento completo do ciclo de vida das lagartas, avaliando-se por meio da densidade de orifícios de saída na superfície dos frutos. Stallion 60 CS (15 $\mathrm{mL})$, Trebon 100 SC (200 mL), Lorsban 480 BR (150 $\mathrm{mL})$, Lannate BR (100 mL), Deltaphos EC (100 mL), Thiobel 500 (250 g), Vertimec 18 CE (100 mL) e Belt (15 mL), em aplicação isolada, resultaram em menor número médio de lagartas que completaram a fase e realizaram o orifício de saída no fruto, diferindo estatisticamente da testemunha e dos tratamentos com Atabron 50 CE (100 mL), Cascade 100 (50 mL), Certero (30 mL), Gallaxy 100 CE (25 mL) e Agree (100 g). Atabron $50 \mathrm{CE}$, Certero, Gallaxy $100 \mathrm{CE}$, Match CE (100 mL), Meothrin $300(50 \mathrm{~mL})$, Pirate $(50 \mathrm{~mL})$ e Agree diferiram estatisticamente da testemunha e entre si, quando aplicados de forma isolada e quando adicionados de óleo vegetal.

Cascade $100(50 \mathrm{~mL})$, Intrepid $240 \mathrm{SC}(50 \mathrm{~mL})$, Rumo GDA (10 g), Akito (50 mL), Orthene 750 BR (100 g) e Cordial $100(75 \mathrm{~mL})$ também reduziram de forma significativa a densidade de orifícios de saída da broca em relação aos frutos da testemunha.

O número médio de lagartas que completaram o ciclo de vida nos frutos imersos em água e em calda com óleo vegetal $(0,25 \%)$ foi de $1,5 \pm 0,1$ e 1,0 $\pm 0,1$ insetos/fruto, respectivamente, diferindo estatisticamente entre si. Assim, a adição do adjuvante também favorece o controle de lagartas em desenvolvimento após alimentação sobre a superfície do fruto com o resíduo seco dos inseticidas, interrompendo o ciclo de vida do inseto e a consequente emergência de adultos (Tabela 4).

Tabela 5 - Eficiência dos inseticidas sobre as lagartas de Neoleucinodes elegantalis em frutos do tomateiro, submetidos ao controle químico em bioensaio de laboratório.

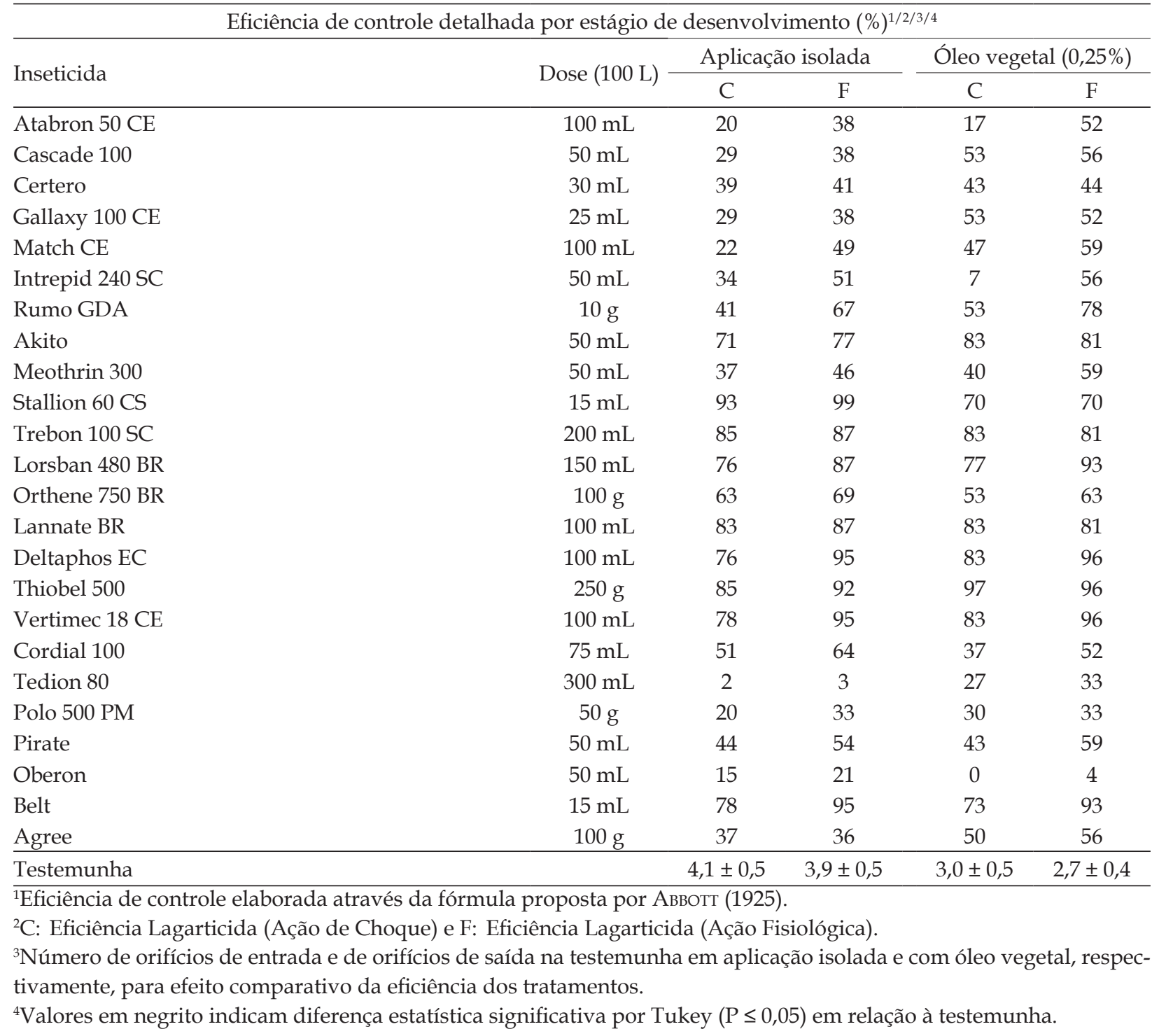


Eficiência de controle/ação larvicida - As ações larvicidas de Rumo GDA (10 g), Cascade 100 (50 $\mathrm{mL})$, Certero (30 mL), Gallaxy $100 \mathrm{CE}(25 \mathrm{~mL})$, Match CE (100 mL) e Agree (100 g) foram significativamente mais efetivas com a adição de óleo vegetal $(0,25 \%)$, sendo os valores $53,53,43,53,47$ e $50 \%$, respectivamente. Os inseticidas que se destacaram em aplicação isolada foram: Stallion 60 CS $(15 \mathrm{~mL})$, Trebon 100 SC (200 mL), Orthene 750 BR (100 g) e Belt (15 mL), em aplicação isolada, com índices de redução de 93, 85, 63 e 78\%, respectivamente, não diferindo dos resultados com adição de óleo vegetal (Tabela 5).

As ações de Atabron 50 CE (100 mL), Certero (30 $\mathrm{mL})$, Gallaxy $100 \mathrm{CE}(25 \mathrm{~mL})$, Match CE $(100 \mathrm{~mL})$, Meothrin $300(50 \mathrm{~mL})$, Pirate $(50 \mathrm{~mL})$ e Agree $(100 \mathrm{~g})$ foram significativamente mais efetivas com adição de óleo vegetal $(0,25 \%)$, sendo da ordem de 52,44 , $52,59,59,59$ e $56 \%$ (Tabela 5), respectivamente. Os inseticidas que se destacaram em aplicação foram: Trebon 100 SC (200 mL), Lannate BR e Belt (15 mL), com índice de redução de lagartas em desenvolvimento de 87,87 e $95 \%$, respectivamente, não diferindo dos resultados encontrados com a aplicação em óleo vegetal. Os produtos em aplicação com óleo vegetal com boa eficiência foram: Lorsban $480 \mathrm{BR}(150 \mathrm{~mL})$, DeltaphosEC $(100 \mathrm{~mL})$, Thiobel500 (250 g)e Vertimec 18 CE (100 mL), com redução no número de orifícios de saída de 93,96, 96 e96\%, respectivamente. Stallion 60 CS resultou na ação fisiológica mais significativa em aplicação isolada, com valor de 99\% (Tabela 5).

Os inseticidas Cascade 100 (50 mL), Intrepid 240 SC (50 mL), Rumo GDA (10 g) e Akito $(50 \mathrm{~mL})$ reduziram em 56, 56, 78 e $81 \%$ a população de lagartas em desenvolvimento quando aplicados de forma isolada, enquanto Orthene 750 BR (100 g) e Cordial $100(75 \mathrm{~mL})$ reduziram em 69 e $64 \%$, respectivamente.

\section{DISCUSSÃO}

A ação de Trebon 100 SC, Lannate BR, Thiobel 500 e Vertimec 18 CE na inviabilização dos ovos de $N$. elegantalis é uma informação estratégica do ponto de vista do manejo da praga na cultura do tomate, pois, a partir do florescimento, GRAVENA; BENVENGA (2003) recomendaram inspeção periódica das plantas com frutos em desenvolvimento para a detecção de ovos, uma vez que os ovos são alvos biológicos favoráveis ao controle químico, devido à permanência temporal na superfície dos frutos durante o período de incubação (RodRIGUES FILHO et al., 2003).

Por outro lado, com a maior ocorrência desse inseto nos cultivos de verão (JORDÃO; NAKANO, 2000; Gravena; Benvenga, 2003), a escolha do inseticida com ação sobre os ovos é decisiva para proteção mais efetiva dos frutos. Tal situação se justifica em função da maior frequência das precipitações (NUNES; LEAL, 2001) que propicia condições climáticas favoráveis ao seu desenvolvimento (MARCANO, 1991a, 1991b), pela maior disponibilidade de hospedeiros alimentares da praga (GALLO et al., 2002), e pela postura realizada diretamente na superfície dos frutos (BLACKMER et al., 2001; Rodrigues Filho et al., 2003; Gravena; BeNVENGA, 2003),

A ação de Thiobel 500 na inviabilização de ovos da broca-pequena-do-frutojá havia sido mencionada por MARQUES et al. (2004), entretanto, o incremento da ação ovicida com a adição de óleo vegetal é uma informação complementar e promissora no manejo da praga em períodos críticos de intensa precipitação. A influência positiva do óleo vegetal pode estar associada à maior permeabilidade do córion do ovo ao inseticida, impedindo o completo desenvolvimento embrionário, pois, segundo MuÑozetal.(1991), ocorre perda de turgescência do córion ao longo do período de incubação, o que resulta em maior resistência à absorção do agrotóxico. A adição de óleo vegetal à calda inseticida com o objetivo de reduzir a tensão superficial da gota para promover maior cobertura dos frutos também foi citada por EIRAS; BLACKMER (2003), pois, devido à alta cerosidade da superfície do fruto, o adjuvante promove cobertura mais uniforme (PAula et al., 1998).

Os orifícios de entrada das lagartas recémeclodidas nos frutos são observados com maior frequência na porção mediana-inferior, indicando que o volume de calda aplicado até atingir o ponto de escorrimento ou a adição de adjuvantes resultaria em cobertura mais homogênea e, consequentemente, maior proteção à infestação por lagartas (EIRAs; BLACKMER, 2003). A informação quanto à preferência de entrada no fruto passa a ser estratégica para o sucesso do manejo da praga, pois permite que o produtor também faça o controle químico com o jato da calda inseticida direcionada para os frutos em fase inicial de desenvolvimento, visando impedir a entrada das lagartas recém-eclodidas.

Os resultados de ação dos produtos no controle da broca-pequena-do-fruto obtidos neste trabalho, por meio dos bioensaios de laboratório, não permitem comparações com as pesquisas de eficácia de inseticidas realizados por LiMA et al. (2001), MARTINELLi et al. (2003) e LEBEDENCO et al. (2007), uma vez que os trabalhos desses autores foram conduzidos em condições de campo. Dentre as razões incluemse que são trabalhos conduzidos em campo, com aplicações sequenciais em intervalos pré-definidos para a avaliação final da densidade de frutos com orifícios de saída das lagartas, não trazendo informações sobre as fases mais vulneráveis da praga (ovos e larvas recém-emergidas). Além disso, os 
trabalhos de eficácia realizados a campo não expressam a importância da avaliação da presença de ovos para o início da aplicação, reforçando o ciclo repetitivo de aplicações sistemáticas a partir do florescimento.

Como já discutido, a informação quanto à preferência de entrada no fruto passa a ser estratégica para o sucesso do manejo da praga, pois permite que o produtor também faça o controle químico com o jato da calda inseticida direcionada para os frutos em fase inicial de desenvolvimento (PAula et al., 1998), visando impedir a entrada das lagartas recémeclodidas. Essa fase de desenvolvimento da praga apresenta curto período de trânsito na superfície dos frutos até localizar o ponto de entrada, passando a se alimentar do epicarpo. A eclosão das lagartas ocorre nas duas primeiras horas da fotofase, entre 6 e $8 \mathrm{~h}$ (EIRAS; BlACKMER, 2003). Portanto, na escolha de inseticidas para o controle da praga, também deve ser contemplada a ação de choque sobre as lagartas recém-eclodidas, o que interrompe o ciclo de vida no ato da alimentação.

Destacaram-se com ação lagarticida: Trebon 100 SC (200 mL), Lannate BR (100 mL), Thiobel 500 (250 g) e Vertimec 18 CE (100 mL), além do Rumo GDA (10 g), Akito $(50 \mathrm{~mL})$, Stallion $60 \mathrm{CS}(15 \mathrm{~mL})$, Lorsban 480 BR (150 mL), Orthene 750 BR (100 g), Deltaphos EC (100 mL), Cordial $100(75 \mathrm{~mL})$ e Belt (15 mL). Os inseticidas Rumo GDA e Thiobel 500 mostraram eficiência de controle significativamente melhores quando adicionados de óleo vegetal, sendo sua potencialização, quando adicionado de óleo mineral $(0,5 \%)$, também relatada por PAULA et al. (1998).

Os pré-requisitos para a escolha do inseticida no manejo da broca-pequena-do-fruto incluem oconhecimento da eficiência sobre as lagartas, particularmente as recém-emergidas. Também, estratégias de seletividade e omprego de inseticidas seletivos para favorecimento do controle biológico aplicado, por exemplo, com T. pretiosum devem ser considerados (Gravena; Benvenga, 2003). Por fim, deve-se atentar para o manejo da resistência, trabalhando com a rotação de inseticidas com distintos mecanismos de ação (Омото, 2000).

\section{CONCLUSÃO}

Os resultados permitem concluir que os inseticidas testados apresentam eficiência de controle, com ação ovicida e larvicida, destacando-se Trebon 100 SC $(200 \mathrm{~mL} / 100 \mathrm{~L})$, Lannate BR $(100 \mathrm{~mL})$, Thiobel $500(250 \mathrm{~g})$ e Vertimec $18 \mathrm{CE}(100 \mathrm{~mL})$; e a adição do adjuvante óleo vegetal $(0,25 \%)$ interfere de forma significativa e positiva na eficiência dos inseticidas sobre os ovos e lagartas de N. elegantalis.

\section{AGRADECIMENTOS}

À Gravena pelo auxílio técnico e financeiro; ao produtor Lázaro Lauro de Andrade pelo uso da área comercial de tomate; aos inspetores de pragas VanderleiSouza Lesse e Eldimar Francisco deSouza, pelo auxílio nas coletas de frutos; e à Fapesp e ao $\mathrm{CNPq}$ pelas bolsas.

\section{REFERÊNCIAS}

ABBOTT, W.S. A method of computing the effectiveness of an insecticide. Journal of Economic Entomology, v.18, p.265-267, 1925.

BLACKMER, J.L.; EIRAS, A.E.; SOUZA, C.L.M. Oviposition preference of Neoleucinodes elegantalis (Guenée) (Lepidoptera: Crambidae) and rates of parasitism by Trichogramma pretiosum Riley (Hymenoptera: Trichogrammatidae) on Lycopersicon esculentum in São José de Ubá, RJ, Brazil. Neotropical Entomology, v.30, p.89-95, 2001.

BRASIL. Ministério da Agricultura, Pecuária e Abastecimento. Agrofit: Sistema de Agrotóxicos Fitossanitários. Disponível em: <http:/ / extranet.agricultura.gov.br/ agrofit>. Acesso em: 15 jan. 2011.

EIRAS A.E.; BLACKMER JL. Eclosion time and larval behavior of the tomato fruit borer, Neoleucinodes elegantalis (Guenée) (Lepidoptera: Crambidae). Scientia Agricola, v.60, p.195-197, 2003.

GALLO, D.; NAKANO, O.; SILVEIRA NETO, S.; CARVALHO R.P.L.; BAPTISTA, G.C.; BERTI FILHO, E.; PARRA, J.R.P.; ZUCCHI, R.A.; ALVES, S.B.; VENDRAMIM, J.D.; MARCHINI, L.C.; LOPES, J.R.S.; OMOTO, C. Entomologia agrícola. Piracicaba: FEALQ, 2002. 920p.

GRAVENA S.; BENVENGA S.R. Manual prático para manejo de pragas do tomate. Jaboticabal: Gravena, 2003. $144 \mathrm{p}$.

JAFFE, K.; MIRÁS, B.; CABRERA, A. Mate selection in the moth Neoleucinodes elegantalis: evidence for a supernormal chemical stimulus in sexual attraction. Animal Behaviour, v.73, p.727-734, 2007.

JORDÃO, A.L.; NAKANO, O. Controle de lagartas dos frutos do tomateiro pelo ensacamento das pencas. Anais da Sociedade Entomológica do Brasil, v.29, p.773-782, 2000.

LEBEDENCO, A.; AUAD, A.M.; KRONKA, S.N. Métodos de controle de lepidópteros na cultura do tomateiro (Lycopersicon esculentum Mill.). Acta Scientiarum Agronomy, v.29, p.339-344, 2007.

LIMA, M.F. de; BOIÇA JUNIOR, A.L.; SOUZA, R.S. de. Efeito de inseticidas no controle da broca pequena 
Neoleucinodes elegantalis na cultura do tomateiro. Revista Ecossistema, v.26, p.54-57, 2001.

MARCANO, R. Estudio de la biologia y algunos aspectos del comportamiento del perforador del fruto del tomate Neoleucinodes elegantalis (Lepidoptera: Pyralidae) en tomate. Agronomia Tropical, v.41, p.257-263, 1991a.

MARCANO, R. Ciclo biologico de perfurador del fruto del tomate Neoleucinodes elegantalis (Guenée) (Lepidoptera: Pyralidae), usando berenjena (Solanum melongena) como alimento. Boletin de Entomologia Venezolana, v.6, p.135-141, 1991b.

MARQUES, L.H. da S; NAKANO, O.; FERREIRA, A.; PERETTO, A.J. Efeito ovicida do Thiobel 500 sobre a broca-pequena do tomateiro, Neoleucinodes elegantalis (Guenée, 1854) (Lepidoptera: Pyralidae) em condições de laboratório. In: CONGRESSO BRASILEIRO DE ENTOMOLOGIA, 20., 2004, Gramado. Resumos. Gramado: SBE. 2004. p.360.

MARTINELLI, S.; MONTAGNA, M.A.; PICINATO, N.C.; SILVA, F.M.A.; FERNANDES, O.A. Eficácia do indoxacarb para o controle de pragas em hortaliças. Horticultura Brasileira, v.21, p.501-505, 2003.

MIRANDA, M.M.M.; PICANÇO, M.C.; ZANUNCIO, J.C.; BACCI, L.; SILVA, E.M. da. Impact of integrated pest management on the population of leafminers, fruit borers, and natural enemies in tomato. Ciência Rural, v.35, p.204-208, 2005.

MUÑOZ, E.; SERRANO, A.; PULIDO, J.I.; DE LA CRUZ, J. Ciclo de vida, hábitos y enemigos naturales de Neoleucinodes elegantalis (Guenée, 1854), (Lepidoptera: Pyralidae), passador del fruto del lulo Solanum quitoense Lam. en el valle del cauca. Acta Agronomica, v.41, p.99104, 1991.
NUNES, M.U.C.; LEAL, M.L.S. Efeito da aplicação de biofertilizante e outros produtos químicos e biológicos, no controle da broca-pequena do fruto e na produção do tomateiro tutorado em duas épocas de cultivo e dois sistemas de irrigação. Horticultura Brasileira, v.19, p.5359, 2001.

OMOTO, C. Modo de ação de inseticidas e resistência de insetos a inseticidas. In: GUEDES, J.C.; COSTA, I.D.; CASTIGLIONI. E. (Ed.). Bases e técnicas do manejo de insetos. Santa Maria: UFSM/CCR/DFS. 2000. p.31-50.

PAULA, S.V. de; PICANÇO, M.C.; FONTES, P.C.R.; VILELA, E.F. Fatores de perdas no tomateiro com adoção de nível de controle e de faixas circundantes. Agro-Ciência, v.14, p.263-271, 1998

PLAZA, A.S.; LEON, E.M.; FONSECA, J.P.; DE LA CRUZ, J. Biology, behaviour and natural enemies of Neoleucinodes elegantalis (Guenée). Revista Colombiana de Entomologia, v.18, p.32-37, 1992.

RODRIGUES FILHO, I.L.; MARCHIOR, L.C.; SILVA, L.V. da. Estudo da viabilidade do ensacamento de pencas em tomateiro tutorado para o controle de Neoleucinodes elegantalis (Guen., 1854) (Lepidoptera: Crambidae) em Paty do Alferes - RJ. Agronomia, v.35, p.33-37, 2001.

RODRIGUES FILHO, I.L.; MARCHIOR, L.C.; SILVA, L.V. da. Análise da oviposição de Neoleucinodes elegantalis (Guén., 1854) (Lep.: Crambidae) para subsidiar estratégia de manejo. Agronomia, v.37, p.23-26, 2003.

Recebido em 27/9/11

Aceito em 9/1/13 